Reading a

cost-effectiveness or

decision analysis study

\title{
Five things to consider
}

\author{
Kate C. Young, PhD \\ Adam G. Kelly, MD \\ Robert G. Holloway, MD
}

\section{Summary}

Cost-effectiveness studies and decision analyses of neurologic practices, treatments, and technologies are increasing in the literature and have an emerging role within both medicine and neurology. Knowledge about these research approaches, how to interpret the results of such studies, as well as an understanding of their limitations will be of growing importance for the practicing neurologist. We discuss 5 aspects of these analyses to increase awareness about the uses and limitations of cost-effectiveness articles in everyday practice.

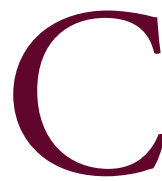

ost-effectiveness research encompasses methods that determine the cost and health-related outcomes of 2 or more competing technologies, interventions, or medications. Decision analysis broadly encompasses similar principles of comparing future, long-term, outcomes from 2 or more alternative regimens. The overarching purpose of cost-effectiveness analyses is to identify strategies that maximize health gains with the resources at hand-or, alternatively, to improve value for money.

The past few years have seen increases in the number of cost-effectiveness publications, primarily in the area of stroke but in other neurologic disciplines as well (figure 1). These research approaches have the potential to assist decision-makers in efficiently allocating resources. Here, we provide an overview of the key considerations in cost-effectiveness research to help the practicing clinician better understand the implications and limitations of this field.

Editorial, page 372 See page 436

Department of Neurology, University of Rochester Medical Center, NY.

Funding information and disclosures are provided at the end of the article. Full disclosure form information provided by the authors is available with the full text of this article at Neurology.org/cp.

Correspondence to: kateyoung524@gmail.com 
Figure 1 Cost-effectiveness publications in neurology

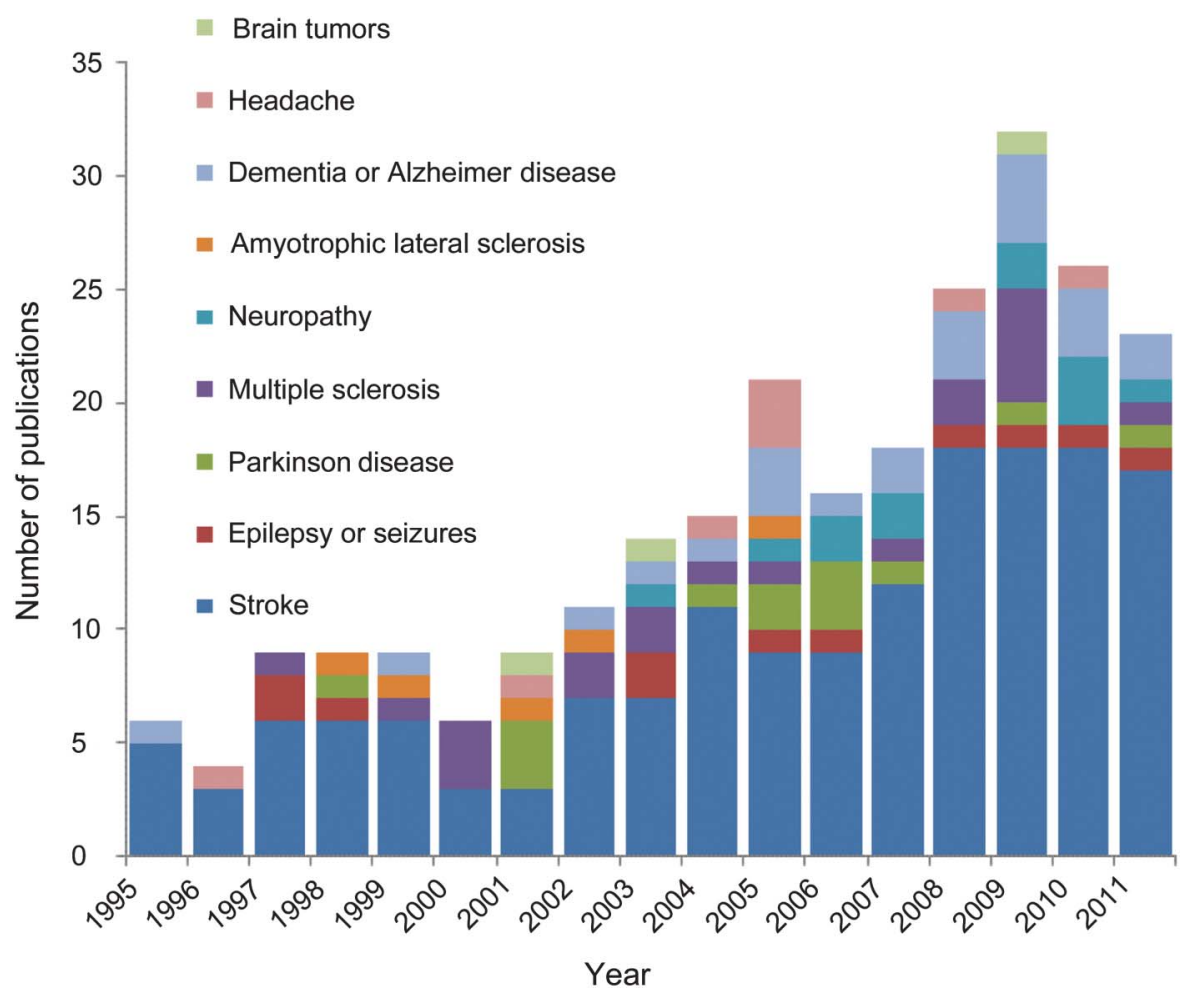

Each year there has been steady growth in the number of cost-effectiveness articles published within neurology subspecialties. Data are from the Cost-effectiveness Analysis Registry. ${ }^{20}$

\section{How are cost-effectiveness studies performed?}

Most research methods can be adapted to address cost-effectiveness questions, including randomized trials and observational research approaches. However, the most common approaches use forms of modeling. With modeling, the goal is to represent the clinical question with sufficient specificity while providing a long enough observation period to capture the most relevant outcomes and costs. Clinical trials and cohorts may not have adequate follow-up or allow for tracking through multiple disease states. For example, to fully capture the health outcomes of a prevention strategy for stroke or a neurodegenerative condition, several years may be needed to effectively capture its preventive benefits in terms of both economic and health outcomes. Oftentimes, clinical trial data or prospective data can be supplemented by assumptions about long-term outcomes that may be less well-established.

There are several different types of analyses. We will use secondary stroke prevention as an example throughout the text comparing standard treatment against a new hypothetical treatment regimen. A cost-effectiveness analysis represents a ratio of financial cost to a unit of measure. An example would be the cost per stroke avoided. For a cost-benefit analysis, both the numerator and the denominator are represented as dollar amounts. The option with the lowest overall cost is the optimal choice. A cost-utility analysis is a specific type of cost-effectiveness analysis where the outcome of the study is a quality-adjusted life-year (or QALY). Such quality adjustment made to life-years gained using utility values (see below) allows for comparisons of value across different interventions and diseases. When the outcomes of competing alternatives are equal (or assumed to be equal), the term cost-minimization analysis is often used as the approach is designed to search for the least costly strategy. Cost-effectiveness is often, though not technically correctly, interchanged with cost-utility analysis.

Costs, utilities, and QALYs Cost data come from a variety of sources including patient diaries, claims information, hospital-level data, clinical trial results, or previously reported 
A cost-effectiveness analysis represents a ratio of financial cost to a unit of measure. An example would be the cost per stroke avoided.

estimates. The sources for cost data should be based on the perspective (see "How do you evaluate the quality of cost-effectiveness studies?") and the time horizon of the model.

A utility is the preference, or a noneconomic value, for a particular health state. Perfect health is anchored at 1 and death is 0 . Negative values, representing states perceived as being worse than death, are also possible. QALYs are the sum of an individual health state's utility multiplied by its duration over the time horizon:

$$
\sum_{k=1}^{n} \text { utility }_{k} * \text { duration }_{k}
$$

For example, 2 years spent in a state with a utility of 0.8 , followed by 6 months in a state with utility 0.4 , would result in 1.8 QALYs $[(2$ years $\times 0.8)+(0.5$ years $\times 0.4)]$. Healthrelated quality of life can be measured using disease-specific surveys or generic surveys (i.e., developed for any patient regardless of disease). While reporting changes in quality of life may itself be informative, to be useful in calculating an incremental cost-effectiveness ratio (ICER), utility values must be available for the survey. Generic quality of life surveys such as the Short Form-36 and EQ-5D are advantageous because they cover multiple aspects of health so that the same survey can be used in persons with stroke or with cancer. The disadvantages to generic quality of life surveys include floor and ceiling effects as well as the potential for limited ability to detect clinically relevant changes in outcomes. In multiple sclerosis (MS), a generic health-related quality of life survey and disease-specific surveys were all reliable but the disease-specific surveys provided additional important information on motor function. ${ }^{1}$ Disease-specific surveys, such as the Stroke-Specific Quality of Life, may be better at representing health-related and clinically relevant changes along a disease spectrum but may lack valid, well-accepted conversions to utility values.

\section{How is the value of a new treatment, technology, or practice defined?}

The result of most cost-effectiveness studies is a ratio of costs to outcomes. An ICER is the net cost of the intervention divided by the net QALYs, or other net effect:

$$
\frac{\left[\$_{\text {intervention }}-\$_{\text {comparator }}\right]}{\left[\mathrm{QALY}_{\text {intervention }}-\mathrm{QALY}_{\text {comparator }}\right]}
$$

The ratio is calculated using the costs and outcomes from an intervention and a comparator. An intervention is the new hypothetical treatment regimen for secondary stroke prevention evaluated against the comparator, or the current standard treatment. Cost-effectiveness analyses can be adapted to include multiple pathways allowing for more than one comparison. Additional steps are required to put the value of this ratio into perspective.

\section{What does it mean to be cost-effective?}

Incremental cost-effectiveness is represented on a 2-dimensional plane with effect, or QALYs, on the $\mathrm{x}$-axis and cost on the $\mathrm{y}$-axis. The costs and outcome data of the new treatment regimen are graphed in 2-dimensional space relative to the current standard treatment, which is placed at the origin. As a result, the plane has 4 quadrants representing the different combinations of relative cost and QALYs (figure 2). Interventions in the southeast quadrant are cost-saving, meaning they have lower cost with greater QALYs. These strategies should be readily adopted 
Figure 2 Cost-effectiveness plane

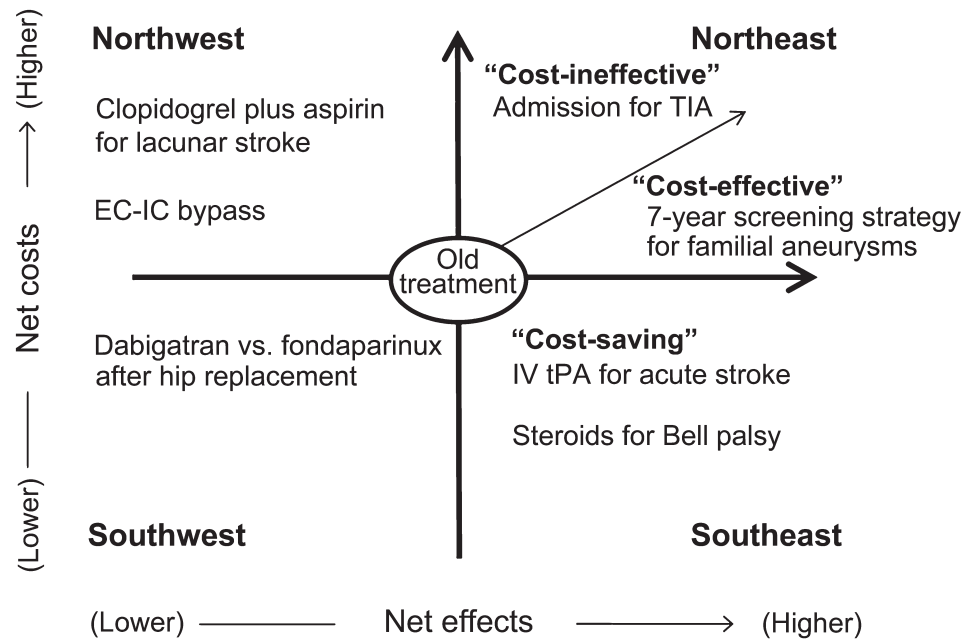

The old or standard treatment (comparator) is placed at the origin of a 2-dimensional graph. The intervention is graphed relative to the comparator based on the incremental effects and costs. ${ }^{2,9,12,21-24}$ tPA $=$ tissue plasminogen activator. Adapted with permission.

into practice. IV tissue plasminogen activator (tPA) for acute ischemic stroke is an example of such a strategy, often referred to as "dominant," which provided greater QALYs and overall financial savings to society. ${ }^{2}$ Treatments in the northwest quadrant are both more costly and less effective. In the southwest quadrant, the new hypothetical treatment regimen would reduce costs at the expense of reduced health. The northeast quadrant represents increased effectiveness of the new treatment regimen but with increased cost. Often, new diagnostic or therapeutic strategies fall into this quadrant and the question becomes how much extra cost is needed to produce the improved health effects.

The threshold to pay more money for health gains varies by country, payer, and perspective (for a definition of perspective, see "How do you evaluate the quality of cost-effectiveness studies?"). Several countries require cost-effectiveness evaluation with (England, Wales: $£ 20,000-30,000$ /QALY) or without (France, Germany, Netherlands, Australia, New Zealand, among others) a threshold. ${ }^{3-6}$ In the United States, a general rule is that ICERs under $\$ 50,000$ per QALY are considered cost-effective, while $\$ 50,000-\$ 100,000$ per QALY (perhaps as high as $\$ 200,000$ for a continuous flow left ventricular assist device as destination therapy in patients with heart failure) is a gray area. ${ }^{7}$

\section{How do you evaluate the quality of cost-effectiveness studies?}

Good research practices for cost-effectiveness studies are available from an internationally recognized joint task force (see reference 8 for the Web link). We should say up front that "all models are wrong, but some are useful." The relevant question is whether or not you can believe the model — does it represent reality with sufficient fidelity that it has face validity? Elements to consider when reading a study are the perspective of the study, the health states represented in the study, the time horizon of the study, evaluating the quality of the data inputs, and how the study addresses uncertainty.

Perspective is the point of view of the analysis and provides part of the relevant framework for the applicability of the study. The societal perspective is the broadest viewpoint, incorporating both direct medical costs and indirect costs such as time lost from work. The payer perspective may include insurance reimbursements or a hospital's viewpoint may include costs or charges.

Time horizon is an important consideration in the review of cost-effectiveness studies. It is the study period over which the model has been designed. An analysis of steroids for Bell palsy 


\section{Cost-effectiveness models are often useful in the decision-making process for formularies, guidelines, and value-based pricing, all of which should be of interest for the practicing clinician.}

had a time horizon of months while the cost-effectiveness of IV thrombolysis (tPA) for acute ischemic stroke was presented at 1 year and over a lifetime. ${ }^{2,9}$ Given the natural history of the disease processes coupled with the scope of the study, one should ensure that the time horizon is sufficient for the disease process.

The type of model influences its applicability. A static model has one transition between health states and one calculation of costs and outcomes over a single prespecified time horizon. Dynamic models allow transitions between health states over time. Costs and outcomes are calculated for each cycle and summed across the total number of cycles. An advantage to dynamic models is the allowance of transitions between health states (e.g., healthy, minor stroke, recovery back to baseline, death).

There are 2 main types of uncertainty with cost-effectiveness analyses: uncertainty about model inputs and uncertainty about the structure of the model..$^{10}$ Uncertainty around data for the inputs can be handled with sensitivity analyses. Each parameter can be individually varied across a prespecified range in a series of one-way sensitivity analyses. For persons with asymptomatic carotid artery stenosis, the cost-effectiveness of carotid endarterectomy compared to medical management was dependent on several factors including sex, age, and annual stroke rate in any territory. ${ }^{11}$ In reality, parameters do not vary in isolation. A probabilistic sensitivity analysis shows how robust the ICER is when parameters are sampled simultaneously.

There may also be uncertainty about the underlying structure or health states of the model itself. Different investigators may construct similar but not identical models for analyzing the same strategy. As an example, it was not cost-effective (ICER > \$millions) to hospitalize someone with a TIA for 48 hours compared to evaluation in a same-day clinic. ${ }^{12}$ However, a 24-hour admission for TIA in another model was cost-effective (ICER $<\$ 60,000 / \mathrm{QALY}){ }^{13}$ Analyses include a finite number of health states usually related to the specific disease process. Cancer may not be included as a health state in a study of a neurologic condition unless there is a known increase in the incidence of cancer with the intervention.

Any decision model requires the researcher (and consequently, the reader) to make certain fundamental assumptions, since even complex models cannot account for all possible clinical scenarios. Authors should attempt to minimize these assumptions when possible, yet be explicit about their rationale when assumptions must be made. Projection of future costs can be problematic given the evolving landscape of health care finance reform.

The study sponsor and other sources of study funding should not be overlooked. In an era of increasing calls for transparency, all funding of authors should be reported beyond what the authors deem is related to the topic. The goal of a commercial enterprise is to promote its specific product and cost-effectiveness analyses can be a means of doing this. Analyses should not be met with abject rejection but with knowledge of the applicability and limitations of the techniques and the ability to draw one's own conclusions.

\section{How do clinicians use cost-effectiveness studies in caring for patients?}

Cost-effectiveness research and decision analyses can play an important role in medical decision-making. As available diagnostic and treatment options for many neurologic conditions 
continue to increase (e.g., noninvasive vascular imaging for stroke and TIA, disease-modifying therapies for MS), there will need to be increased use of research that analyzes not just effectiveness but comparative effectiveness of these various strategies. Cost-effectiveness studies can help identify strategies that provide the best value for both patients and society (better outcomes at lower cost); in addition, they can identify options that provide very little value, ones that patients and families should be counseled to avoid. In this manner, cost-effectiveness research may have a major role within contemporary initiatives

\section{Reading a cost-effectiveness or decision analysis study: Five things to consider}

- How are cost-effectiveness studies performed?

- How is the value of a new treatment, technology, or practice defined?

- What does it mean to be cost-effective?

- How do you evaluate the quality of costeffectiveness studies?

- How do clinicians use cost-effectiveness studies in caring for patients? such as the "Choosing Wisely" campaign and the concept of parsimonious medicine, which seek to identify management strategies that maximize value and eliminate or minimize those associated with unintended harm (Web link to the American Academy of Neurology's "Choosing Wisely" page: http://www. aan.com/go/practice/quality/choosingwisely). ${ }^{14,15}$ While some fear that comparative effectiveness research may serve as a precursor to "rationing" of medical services, using these types of studies, which seek to maximize value and minimize waste, should be preferred over unguided decisions regarding care. ${ }^{16}$

To some degree, decision analysis can also account for a major factor in medical decision-making-patient and family preferences - which is not typically addressed by other clinical trial or observational research methods. For example, a decision model may reveal that a certain strategy is the preferred option only if the patient would value the resultant health state at or above a certain utility. ${ }^{17}$ While utility values are a methodologic research construct, they may still provide a valuable framework when discussing various treatment options and the anticipated value of expected outcome states with patients and families.

Cost-effectiveness models are often useful in the decision-making process for formularies, guidelines, and value-based pricing, all of which should be of interest for the practicing clinician. The United States has no explicit role for the use of cost-effectiveness analysis in decision-making; however, insurance coverage may be limited for items in the northwest quadrant. A more concrete example of resource allocation is preauthorization or lack of coverage for certain medical tests or procedures. The National Institute for Health and Clinical Excellence (England and Wales) solicits information on how the new technology or drug provides value for money-Is it cost-effective? Canada has a system whereby cost-benefit analyses are carried out for considerable regulatory proposals with the purpose of maximizing net economic and other relevant benefits, including social benefits, to the greater society. Analysis of these data during the review process can lead to delayed approvals or limited indications for certain treatments, such as with glargine insulin in Canada and Australia. ${ }^{18}$

It remains to be seen how Patient Protection and Affordable Care Act directives will ultimately affect coverage. While the Patient Protection and Affordable Care Act tries to promote value-based health care within the confines of finite resources, it must do so without establishing a threshold for cost-effective or recommended care (Section 1182, e). Nonetheless, resource allocation does indeed exist and providers should have some understanding of how these decisions are made by payers. Value-based health care should focus on optimizing health care and its delivery with definitive outcome measures as well as tools that allow for comparisons across other disease states. As value in medicine and cost-effectiveness are integrated in the future, the next generation of physicians, including neurologists, will need to be more familiar with the place of such analyses in decision-making, insurance coverage, public policy, and legislation. ${ }^{19}$ 


\section{REFERENCES}

1. Vickrey BG, Hays RD, Genovese BJ, Myers LW, Ellison GW. Comparison of a generic to diseasetargeted health-related quality-of-life measures for multiple sclerosis. J Clin Epidemiol 1997;50:557-569.

2. Fagan SC, Morgenstern LB, Petitta A, et al. Cost-effectiveness of tissue plasminogen activator for acute ischemic stroke: NINDS rt-PA Stroke Study Group. Neurology 1998;50:883-890.

3. Claxton K, Briggs A, Buxton MJ, et al. Value based pricing for NHS drugs: an opportunity not to be missed? BMJ 2008;336:251-254.

4. Boersma C, Broere A, Postma MJ. Quantification of the potential impact of cost-effectiveness thresholds on Dutch drug expenditures using retrospective analysis. Value Health 2010;13:853-856.

5. Lopert R. Evidence-based decision-making within Australia’s Pharmaceutical Benefits Scheme. In: Commonwealth Fund [serial online] 2009;60:1-7. Available at: http://www.commonwealthfund.org/ /media/ Files/Publications/Issue\%20Brief/2009/Jul/Chalkidou/1297_Lopert_CER_Australia_issue_brief_724.pdf. Accessed April 15, 2013.

6. Simoens S. Health economic assessment: cost-effectiveness thresholds and other decision criteria. Int J Environ Res Public Health 2010;7:1835-1840.

7. Rogers JG, Bostic RR, Tong KB, Adamson R, Russo M, Slaughter MS. Cost-effectiveness analysis of continuous-flow left ventricular assist devices as destination therapy. Circ Heart Fail 2012;5: $10-16$.

8. Joint Modeling Good Research Practices Task Force. Available at: http://www.ispor.org/taskforces/ GRPModelingTf.asp. Accessed April 15, 2013.

9. Hernandez RA, Sullivan F, Donnan P, Swan I, Vale L. Economic evaluation of early administration of prednisolone and/or aciclovir for the treatment of Bell's palsy. Fam Pract 2009;26:137-144.

10. Briggs AH. Handling uncertainty in cost-effectiveness models. Pharmacoeconomics 2000;17: 479-500.

11. Thapar A, Garcia ML, Epstein D, Shalhoub J, Davies AH. Modelling the cost-effectiveness of carotid endarterectomy for asymptomatic stenosis. Br J Surg 2013;100:231-239.

12. Joshi JK, Ouyang B, Prabhakaran S. Should TIA patients be hospitalized or referred to a same-day clinic?: a decision analysis. Neurology 2011;77:2082-2088.

13. Nguyen-Huynh MN, Johnston SC. Is hospitalization after TIA cost-effective on the basis of treatment with tPA? Neurology 2005;65:1799-1801.

14. Tilburt JC, Cassel CK. Why the ethics of parsimonious medicine is not the ethics of rationing. JAMA 2013;309:773-774.

15. Cassel CK, Guest JA. Choosing wisely: helping physicians and patients make smart decisions about their care. JAMA 2012;307:1801-1802.

16. Mushlin AI, Ghomrawi H. Health care reform and the need for comparative-effectiveness research. N Engl J Med 2010;362:e6.

17. Kelly AG, Holloway RG. Health state preferences and decision-making after malignant middle cerebral artery infarctions. Neurology 2010;75:682-687.

18. Clement FM, Harris A, Li JJ, Yong K, Lee KM, Manns BJ. Using effectiveness and costeffectiveness to make drug coverage decisions: a comparison of Britain, Australia, and Canada. JAMA 2009;302:1437-1443.

19. Holloway RG, Ringel SP. Getting to value in neurological care: a roadmap for academic neurology. Ann Neurol 2011;69:909-918.

20. Center for the Evaluation of Value and Risk in Health. Available at: https://research.tufts-nemc.org/cear $4 /$. Accessed May 31, 2013.

21. Failure of extracranial-intracranial arterial bypass to reduce the risk of ischemic stroke: results of an international randomized trial: The EC/IC Bypass Study Group. N Engl J Med 1985;313: 1191-1200.

22. Benavente OR, Hart RG, McClure LA, Szychowski JM, Coffey CS, Pearce LA. Effects of clopidogrel added to aspirin in patients with recent lacunar stroke. N Engl J Med 2012;367:817-825.

23. Bor AS, Koffijberg H, Wermer MJ, Rinkel GJ. Optimal screening strategy for familial intracranial aneurysms: a cost-effectiveness analysis. Neurology 2010;74:1671-1679.

24. Dabigatran etexilate for the prevention of venous thromboembolism after hip or knee replacement surgery in adults. In: Agency for Healthcare Research and Quality, 2008. Available at: http:// guideline.gov/content.aspx?id=13169. Accessed April 15, 2013.

\section{STUDY FUNDING}

Dr. Young is funded by the Greater Rochester Health Foundation and by the University of Rochester Departments of Neurology and Neurosurgery. Dr. Holloway is funded by the NIH. 


\section{DISCLOSURES}

K. Young receives funding from the Greater Rochester Health Foundation. A. Kelly receives funding from the Donald W. Reynolds Foundation. R. Holloway is an Associate Editor of Neurology Today, a consultant to Milliman Guidelines, and receives funding from the NIH. Full disclosure form information provided by the authors is available with the full text of this article at Neurology.org/cp.

\section{Related articles from other AAN physician and patient resources}

Neurology ${ }^{\circledR} \quad \bullet \quad$ www.neurology.org

The American Academy of Neurology's Top Five Choosing Wisely recommendations September 10, 2013;81:1004-1011.

Cost-effectiveness of apixaban vs warfarin for secondary stroke prevention in atrial fibrillation

October 2, 2012;79:1428-1434.

Cost-effectiveness of disease-modifying therapy for MS: A population-based study July 26, 2011;77:355-363.

\section{Continuum ${ }^{\circledR} \quad$ - www.ContinuumJournal.com}

Should the Cost of Care For Patients With Glioblastoma Influence Treatment Decisions? April 2012;416-420.

\section{Neurology Now ${ }^{\circledR}$ - www.neurologynow.com}

Choosing Wisely: Money spent on unnecessary medical services does nothing to improve human health April/May 2013:9:6.

Choosing Wisely: Don't Waste Your Money on Unnecessary Medical Care April/May 2013;9:23-25.

\section{Neurology Today ${ }^{\circledR} \quad$ - www.neurotodayonline.com}

When Patients Equate Cost with Best Medical Care: What Neurologists Can Do to Help Them Choose Wisely April 4, 2013;13:20-22.

Choosing Wisely: 5 Things Neurologists and Patients Should Question February 21, 2013;13:1,22-24. 